\title{
Macroscopic motion and gravitation in thermodynamics
}

\section{Juan R Sanmartín}

ETS Ingenieros Aeronáuticos, Universidad Politécnica, 28040 Madrid, Spain

Received 26 July 1994

\begin{abstract}
A series of examples rarely presented to students is discussed to illustrate a property of thermodynamic equilibrium: small parts of a fully isolated system move as if points of a rigid body, so as to minimize the macroscopic (kinetic) energy $E_{\mathrm{M}}$. Most examples lie in the fields of astronomy and astrophysics, $E_{\mathrm{M}}$ then including the gravitational energy. The paradoxical behaviour of gravitation, in particular in the extreme case of black holes, is discussed.
\end{abstract}

\section{Introduction}

Very small but still macroscopic parts of a fully isolated system in thermal equilibrium move as if points of a rigid body (Landau and Lifshitz 1969). This is a consequence of the fact that maximum entropy requires maximum internal energy $E_{1}$, leading to minimum macroscopic kinetic energy $E_{\mathrm{M}}$, which turns out to be a characteristic of rigid-body motion (for given momentum and angular momentum). As recently recalled by Diu et al (1990), this definite aspect of equilibrium is rarely touched upon in thermodynamic texts, even though energy 'dissipation' is often acknowledged as part of the approach to equilibrium.

In this paper, several examples of the minimum$E_{\mathrm{M}}$ property are discussed in a unified manner. Since this property clearly holds for a full macroscopic energy $E_{\mathrm{M}}$ when it includes a long-range interaction, usually gravitation, most examples lie in the fields of astronomy and astrophysics, where the thermodynamic emphasis, however, is often lost. Further, the very long range of gravitation, which is essential for its energy to enter $E_{\mathrm{M}}$, makes it a non-extensive quantity and leads to paradoxical thermodynamic behaviour, e.g. negative heat capacity, which, again, students are rarely introduced to (Grandy 1987).

\section{Macroscopic motion at equilibrium}

For the simplest systems, the fundamental relation
Resumen. Se discuten una serie de ejemplos -raramente expuestos en textos termodinámicos- a fin de ilustrar la siguiente propiedad: Cualquier sistema en equilibrio se mueve como un cuerpo rígido, minimızando así la energía macroscópica (cinética) $E_{\mathrm{M}}$. En su mayoría, los ejemplos provienen de la astronomía o la astrofísica, en cuyo caso $E_{M}$ incluye la energía gravitatoria Se discute el paradójico comportamiento termodinámico de la gravitación, en particular en el caso extremo de un agujero negro

determining thermodynamic behaviour gives entropy as a function $S\left(N, V, E_{\mathrm{i}}\right)$ of number of particles $N$, volume $V$, and internal energy, the temperature being $T \equiv \partial E_{1} /\left.\partial S\right|_{N, V}>0$. For any small but macroscopic part $\alpha$ of the whole system, one may introduce its entropy $\delta S_{\alpha} \equiv S\left(\delta N_{\alpha}, \delta V_{\alpha}, \delta E_{1 \alpha)}\right.$. Since $T$ is uniform at equilibrium, a maximum in the entropy $S \equiv \sum_{\alpha} \delta S_{\alpha}$ of an isolated body, with all $\delta N_{\alpha}$ given, requires maximum $E_{z} \equiv \sum_{\alpha} \delta E_{1 \alpha}$, and thus minimum macroscopic kinetic energy

$$
E_{\mathrm{M}}=\sum_{\alpha} \delta P_{\alpha}^{2} / 2 m \delta N_{\alpha}
$$

at fixed values of total momentum $\sum_{\alpha} \delta \vec{P}_{\alpha}$ and angular momentum $\sum_{\alpha} \bar{r}_{\alpha} \wedge \delta \vec{P}_{\alpha} \equiv \bar{L}_{0} ; m$ is the particle mass and $\bar{r}_{\alpha}$ is the radius vector for part $\alpha$. An equivalent condition is that the energy

$$
\frac{\delta P_{\beta}^{2}}{2 m \delta N_{\beta}}+\frac{\delta P_{\gamma}^{2}}{2 m \delta N_{\gamma}},
$$

for any distinct parts $\beta$ and $\gamma$, be minimum at fixed values of $\delta \vec{P}_{\beta}+\delta \bar{P}_{\gamma}$ and $\vec{r}_{\beta} \wedge \delta \bar{P}_{\beta}+\bar{r}_{\gamma} \wedge \delta \bar{P}_{\gamma}$; note that this energy is now just a function of, say, $\delta P_{\beta \Perp}$ (momentum parallel to $\vec{r}_{\gamma}-\tilde{r}_{\beta}$ ). The minimum then occurs at

$$
\delta P_{\gamma \|} / m \delta N_{\gamma}=\delta P_{\beta \|} / m \delta N_{\beta},
$$

which can be read as ' $\delta \bar{P}_{\alpha} / m \delta N_{\alpha}-\bar{\omega} \wedge \bar{r}_{\alpha}$ independent of $\alpha^{3}$. This is rigid-body kinematics (with angular velocity $\bar{\omega}$ constant in time).

As a surprisingly simple example, consider a block 1 


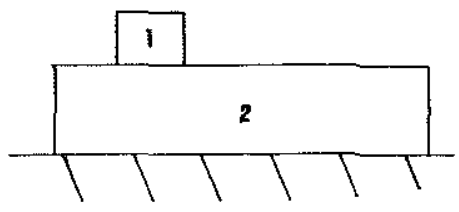

(a)

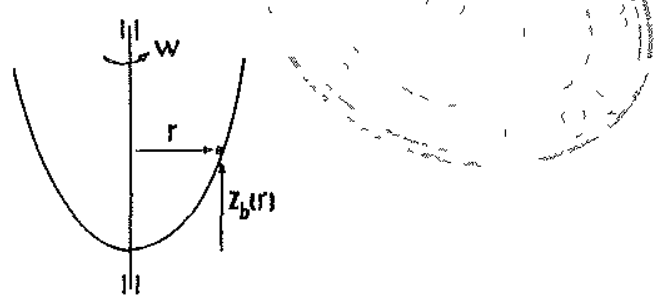

(b)

Figure 1. Thermodynamic equilibrium for macroscopic motion with conserved (a) momentum, and (b) angular momentum.

sliding over another block 2 , itself sliding without friction on a horizontal plane (figure 1(a)). If air drag is negligible, total horizontal momentum is conserved. For a general interaction between the blocks, thermodynamic equilibrium would require minimum macroscopic energy, yielding single rigid-body motion. Indeed, for minimum

$$
E_{\mathrm{M}} \equiv \frac{1}{2} M_{1} v_{1}^{2}+\frac{1}{2} M_{2} v_{2}^{2}
$$

subject to the condition $M_{1} v_{1}+M_{2} v_{2}=$ constant, we obtain

$$
\mathrm{d} E_{\mathrm{M}} / \mathrm{d} v_{1}=M_{1} v_{1}+M_{2} v_{2} \mathrm{~d} v_{2} / \mathrm{d} v_{1}=0 \rightarrow v_{2} \approx v_{1},
$$

with $\mathrm{d}^{2} E_{\mathrm{M}} / \mathrm{d} v_{1}^{2}>0$. Clearly, any friction law that keeps acting as long as there is relative sliding, incorporates thermodynamic finality and gives the same result.

The time required to reach equilibrium can affect the dynamics. A ball, if dropped, will come to rest on the floor, at minimum $E_{\mathrm{M}}$; previously, however, the ball may bounce a large number of times if the duration of contact at each bounce is short compared with the duration of the thermodynamic process. A system may be trapped in metastable motions (corresponding to relative entropy maxima) for very long times.

Consider a particle of mass $M$ sliding inside a bowl of shape $z_{\mathrm{b}}(r)$ that rotates freely around its fixed, vertical axis (figure $1(\mathrm{~b})$ ), the corresponding moment of inertia being $I$. At equilibrium,

$$
E_{\mathrm{M}}=\frac{1}{2} I \omega^{2}+\frac{1}{2} M\left[v_{\theta}^{2}+v_{r}^{2}+v_{r}^{2}\left(\frac{\mathrm{d} z_{\mathrm{b}}}{\mathrm{d} r}\right)^{2}\right]+M g z_{\mathrm{b}}(r)
$$

must be minimum subject to condition $I \omega+M v_{\theta} r \equiv L_{0} ;$ the minimum occurs at $v_{r}=0$, $v_{\theta}=\omega r=r L_{0} /\left(I+M r^{2}\right), r$ being given, for a broad class of shapes, by

$$
\frac{\mathrm{d} z_{b}}{\mathrm{~d} r}=\frac{r L_{0}^{2}}{g\left(I+M r^{2}\right)^{2}}
$$

On the other hand, a direct mechanical analysis, using dry friction, allows any equilibrium within a $r$-range (determined by the friction coefficient) that includes the value for maximum entropy, as given by (1). The dry fiction law is thus thermodynamically peculiar. This is because a solid can support shear stresses (and strains) in metastable equilibria, for times that may be considered infinite; $S$ is then allowed to depend on $N, E_{z}$ and a number of strain or stress variables, rather than just $V$, or pressure $p$ (Callen 1960). Note that, for mechanical equilibria other than that determined by (1), the particle of figure 1(b) does exert a shear stress on the bowl.

A well known application of the $E_{\mathrm{M}}$-principle concerns an isolated solid body. Being incompatible with precession, a constant angular velocity must lie along a principal axis of inertia; at equilibrium one has $E_{\mathrm{M}}=L_{0}^{2} / 2 I$, with $I$ the largest among the three principal moments. In general, a rotating body will deform so as to increase its largest moment. For bodies large enough, self-gravitation has clearly an opposite effect. A classic problem, the equilibrium figure of a rotating, self-gravitating liquid mass, exhibits a rich variety of solutions; slow dissipative effects, taking the system from one figure to another with lower $E_{\mathrm{M}}$, gave origin in the past to the concept of 'secular' instability, so familiar in astrophysics (Lamb 1932).

\section{Gravitation}

Consider here a simpler problem: a planet-satellite system, both spin axes being perpendicular to the orbital plane. At equilibrium,

$$
E_{\mathrm{M}} \equiv \frac{1}{2} I_{\mathrm{s}} \omega_{\mathrm{s}}^{2}+\frac{1}{2} I_{\mathrm{p}} \omega_{\mathrm{p}}^{2}-\frac{1}{2} \frac{G M_{\mathrm{p}} M_{\mathrm{s}}}{a(\hat{\Omega})}
$$

must be minimum subject to condition

$$
I_{\mathrm{s}} \omega_{\mathrm{s}}+I_{\mathrm{p}} \omega_{\mathrm{p}}+\frac{G M_{\mathrm{p}} M_{\mathrm{s}}}{a(\hat{\Omega}) \hat{\Omega}}\left(1-e^{2}\right)^{\mathrm{l} / 2}=L_{0},
$$

where $e$ is the eccentricity, $I_{p}$ and $I_{s}$ the largest principal moments of inertia, $\hat{\Omega}$ the mean orbital angular velocity, and $a$ the major semi-axis, related to $\Omega$ by

$$
a^{3}=G\left(M_{\mathrm{p}}+M_{\mathrm{s}}\right) / \hat{\Omega}^{2} .
$$




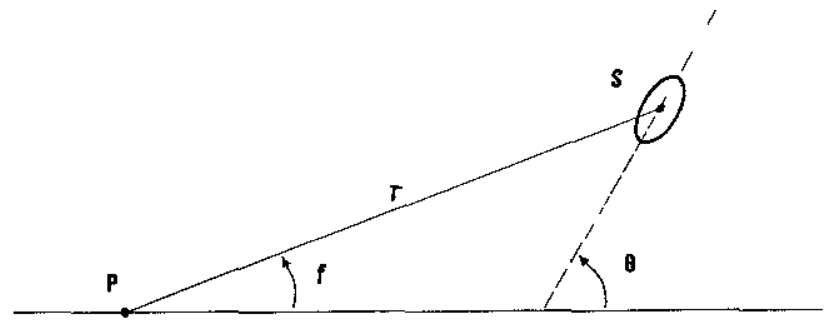

Figure 2. Geometry of spin-orbit interaction for a satellite $S$ with unequal principal moments of inertia; $f$ is the true anomaly, $\theta$ the angular position of the long axis, and $r$ the distance to planet $P$.

Energy is dissipated in the motion produced by tidal (time and space dependent, gravitational) forces. Minimum $E_{\mathrm{M}}$ may be shown to occur at

$$
e=0, \quad \hat{\Omega}=\omega_{\mathrm{p}}=\omega_{\mathrm{s}} \text { (dual synchronism). }
$$

An example of such equilibrium has been found recently: Pluto and its satellite Charon move fully as a single rigid body, the periods for circular revolution, and planet and satellite spins, all three being 6.387 days! (Binzel 1990).

Pluto and Charon have comparable masses and sizes. Usually, however, small ratios $I_{\mathrm{s}} / M_{\mathrm{s}} a^{2}, I_{\mathrm{s}} / I_{\mathrm{p}}$ (and $M_{\mathrm{s}} / M_{\mathrm{p}}$ ) allow a value $\mathrm{d} \omega_{\mathrm{s}} / \mathrm{d} t$ much larger than $\mathrm{d} \omega_{\mathrm{p}} / \mathrm{d} t$ and $\mathrm{d} \hat{\Omega} / \mathrm{d} t$ in equation (3), and the satellite spin first reaches synchronism with a frozen orbital motion. The spin dynamics is then governed by a standard equation (Goldreich and Peale 1966),

$$
I_{\mathrm{s}} \ddot{\theta}+\frac{3}{2}\left(B_{\mathrm{s}}-A_{\mathrm{s}}\right) \frac{G M_{\mathrm{p}}}{r^{3}} \sin 2(\theta-f)=0,
$$

where $f$ is the true anomaly, $\theta$ is the angular position of the long axis (figure 2) and $A_{\mathrm{s}}$ and $B_{\mathrm{s}}\left(>A_{\mathrm{s}}\right)$ are the lowest two principal moments of inertia. Typically, permanent deviations from axial symmetry, though small for large satellites $\left(B_{\mathrm{s}}-A_{\mathrm{s}} \equiv \epsilon^{2} I_{\mathrm{s}} \simeq 2 \times\right.$ $10^{-4} I_{\mathrm{s}}$ for the Moon), dominate the changing tidal distortions, which are not included in equation (5). If $e$ vanishes we have $r=a, G M_{\mathrm{p}} / a^{3}=\hat{\Omega}^{2}, f=$ constant $\equiv \hat{\Omega}$, making (5) a conservative pendulum equation for $\gamma \equiv 2(\theta-f)$; clearly, adding a small tidal dissipation would change an initial circulation, usually fast $\left(\left|\dot{\gamma}_{0}\right| \sim \hat{\Omega} \gg\right.$ natural frequency $\equiv$ $\left.3^{1 / 2} \epsilon \hat{\Omega}\right)$, to libration, then to rest at minimum energy $E_{\mathrm{M}}$, which occurs at synchronous spin $\left(\omega_{\mathrm{s}} \equiv\right.$ $\dot{\theta}=\hat{\Omega}, \theta=f$ ).

If $e$ does not vanish, full synchronism is impossible. The satellite, however, may reach some metastable equilibrium, at a relative $E_{\mathrm{M}}$ minimum (entropy maximum). An averaging scheme may be shown to transform equation (5) into a new pendulum equation for $\gamma_{n} \equiv 2 \theta-n \hat{\Omega} t$, whenever dissipation itself leads to condition $\dot{\theta} \simeq n \hat{\Omega} / 2$, with $n$ any integer (spin-orbit resonance); the natural frequency is now $3^{1 / 2} \epsilon \hat{\Omega} \times h_{n}(e)$, the function $h_{n}(e)$ being such that $h_{n}(0)=0$ for $n \neq 2$ and $h_{2}(0)=1$ (Goldreich and Peale 1966). Then, if trapped in libration, a satellite will again end at 'rest' $\left(\gamma_{n}=0, \dot{\gamma}_{n}=0\right)$. Over 20 satellites have been found in the 'synchronous', $n=2$ resonance; Mercury, as a Sun's satellite with $e \simeq 0.206$, is in the $n=3$ resonance, its revolution and spin periods being 87.969 and 58.646 days respectively.

Actually, residual tides due to variations of radial distance in these $e \neq 0$, metastable equilibria, will destroy them, driving the eccentricity to zero and the spin to truly synchronous. If a primary, however, has more than one satellite, an orbital resonance (two satellite revolution periods being in the ratio of small integers) can keep an eccentricity finite. 'This is the case with Saturn's Hyperion, its period being $\frac{4}{3}$ that of Titan. As a consequence, Hyperion, which is small and thus highly aspherical $(\epsilon \simeq 0.51)$, exhibits chaotic, rather than synchronous, spin dynamics (Wisdom et al 1984).

Similarly, if the orbital plane is fixed (a condition assumed above), tides may be shown to bring the satellite equator to that plane as $e \rightarrow 0$ and $\omega_{\mathrm{s}} \rightarrow \hat{\Omega}$. The orbital plane, however, must keep precessing if initially inclined with respect to some nearly 'invariable' plane (the ecliptic, in the case of the Moon). Tidal evolution then ends in a 'Cassini' state, spin axis and orbit normal precessing about the normal to the invariable plane, keeping rigidly coplanar, as observed by G D Cassini for the Moon at the end of the seventeenth century.

We might expect that including Titan in the tidal study of the Saturn-Hyperion system, say, would allow both satellites to reach rigid-body equilibrium with their primary, through slower dissipation processes. In fact, Thomson and Tait (1962) suggested just that for the Sun-Earth-Moon system. If necessary, one could go on including more satellites, and planets, trying to avoid any obstacles to equilibrium, but the times involved soon become fantastically larger than the age of the Universe. A deeper difficulty, anyway, stems from the special thermodynamic character of gravitation.

\section{Thermodynamic paradoxes of gravitation}

Assume that values $e \simeq 0$ and $\omega_{\mathrm{s}} \simeq \hat{\Omega}$ in equations; 

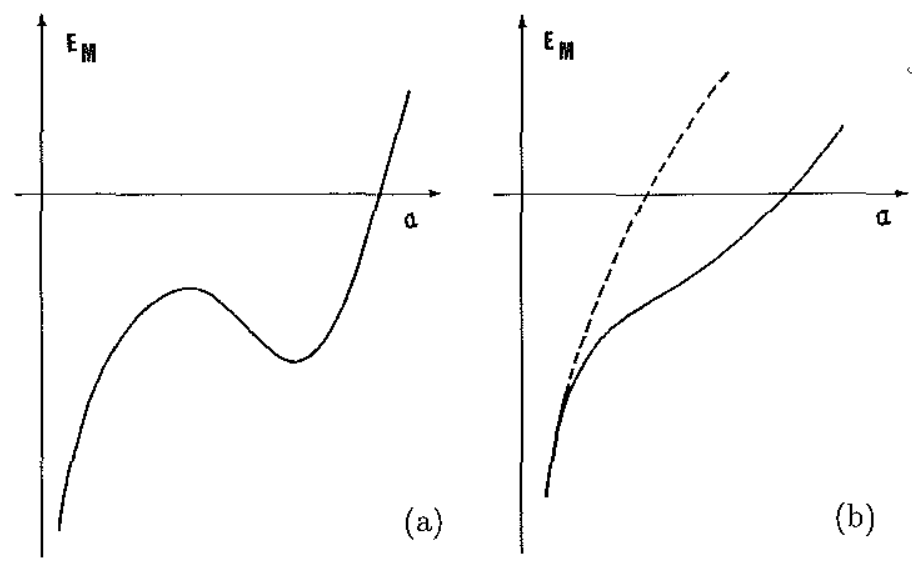

Figure 3. Macroscopic energy $E_{M}$ versus major semiaxis a in equations $(2)-(4)$, with $I_{\mathrm{s}}=0$ and $e=0^{\cdot}$ (a) $\hat{\Omega}>0$ and $L_{0}>4\left(G M_{\mathrm{p}}^{2} M_{\mathrm{s}}^{2} I_{\mathrm{p}}^{3} / 27\right)^{1 / 4} ;$ (b) $\hat{\Omega}<0$ (broken curve), $\hat{\Omega}>0$ and $L_{0}<4\left(G^{2} M_{\mathrm{p}}^{2} M_{\mathrm{s}}^{3} I_{\mathrm{p}} / 27\right)^{1 / 4}$ (full curve).

(2)-(4) have indeed been reached, neglect $I_{\mathrm{s}}$ against $M_{\mathrm{s}} a^{2}$, and $M_{\mathrm{s}}$ against $M_{\mathrm{p}}$, and take $L_{0}>0$ (with no loss of generality). This yields a function $E_{\mathrm{M}}(a)$ that presents one minimum and one maximum (both exhibiting rigid-body motion, i.e. $\omega_{\mathrm{p}}=\hat{\Omega}$ ) for $\hat{\Omega}>0$ and $L_{0}>L_{*} \equiv 4\left(G^{2} M_{\mathrm{p}}^{2} M_{\mathrm{s}}^{3} I_{\mathrm{p}} / 27\right)^{1 / 4}$ (figure $3(\mathrm{a})$ ). The figure applies reasonably well to the Moon, which is receding from Earth, moving from $a(\max )$ to $a(\min )$ (Darwin 1880). Note, however, that the equilibrium at $a(\mathrm{~min})$ is metastable, the $E_{\mathrm{M}^{-}}$ minimum being relative: to the left of the maximum the entropy increases indefinitely with decreasing $a$. Further, if $L_{0}$ is less than $L_{*}$, or $\hat{\Omega}$ is negative (and $\omega_{\mathrm{p}}$ positive), $E_{\mathrm{M}}(a)$ presents no extrema at all (figure 3(b)); no entropy maximum exists, and no rigid-body motion is possible, the satellite nccessarily falling upon the planet. This is the case of Triton, which revolves around Neptune opposite the 'normal', counterclockwise rotation of the planet, upon which is indeed collapsing. As confirmed later, a runaway in entropy is a common fate of gravitationally bound systems.

For artificial satellites, $M_{\mathrm{s}}$ is comparatively very small; w1th $L_{0} \gg L_{*}$ and an initial planetary spin $\omega_{\mathrm{p} 0} \simeq L_{0} / I_{\mathrm{p}}, a(\min )$ and $a(\max )$ in figure $3(\mathrm{a})$ take simple forms,

$$
\begin{aligned}
& a(\max ) \simeq\left(\frac{G M_{\mathrm{p}}}{\omega_{\mathrm{p} 0}^{2}}\right)^{1 / 3}, \\
& a(\min ) \simeq \frac{\left(I_{\mathrm{p}} / M_{\mathrm{s}}\right)^{2}}{G M_{\mathrm{p}} / \omega_{\mathrm{p} 0}^{2}} .
\end{aligned}
$$

For the Earth, $a(\max )$ is the radius of a geostationary orbit, and $a(\mathrm{~min})$ is much larger than the visible Universe, even for 'big' satellites (e.g., the Space Station Mir)! Consequently, $E_{\mathrm{M}}(a)$ shows only a maximum, dissipation always moving satellites away from geostationary positions. The rate of dissipation is, naturally, negligible except at a low orbit, where it is caused by air drag; this emphasizes the thermodynamic character of the end state for equations (2)-(4): tidal forces and air drag are just ktnetic mechanisms. A space tether (a wire, millimetre thin and tens of kilometres long, tied to a satellite in the ionosphere and kept vertical by gravity-gradient forces) uses another kinetic mechanism: the geomagnetic field induces a current and brakes the satellite, taking power into ohmic, radiation and tetherionosphere contact impedances (Sanmartín et al 1993).

Satellite decay at low Earth orbit (left of maximum in figure 3(a)) shows a second peculiar aspect of gravitation. Equations (2)-(4), with $e=0$ and $I_{\mathrm{s}} / M_{\mathrm{s}} a^{2}$ and $M_{\mathrm{s}} / M_{\mathrm{p}}$ negligible, give

$$
\begin{aligned}
& \frac{\mathrm{d} E_{\mathrm{M}}}{\mathrm{d} t}=\frac{\hat{\Omega}}{\hat{\Omega}-\omega_{\mathrm{p}}} \frac{\mathrm{d} E_{\mathrm{orb}}}{\mathrm{d} t} \\
& \left(E_{\mathrm{orb}}=-\frac{G M_{\mathrm{p}} M_{\mathrm{s}}}{2 a}\right) .
\end{aligned}
$$

Since the orbital kinetic energy is the opposite of total orbital energy $E_{\mathrm{O} 1 \mathrm{~b}}\left[\frac{1}{2} M_{\mathrm{s}} a^{2} \hat{\Omega}^{2}=G M_{\mathrm{p}} M_{\mathrm{s}} / 2 a\right.$, equation (4)], which decreases with $E_{\mathrm{M}}$, air drag, in a sense, results in a gain speed, the so called satellite 'paradox' (Mills 1959). A variant of this thermodynamic paradox is well known in astrophysics. The virial theorem for a self-gravitating gas system shows that global values of internal (kinetic) energy $E_{1}$, and total energy $E \equiv E_{1}+E_{\mathrm{g}}\left(E_{\mathrm{M}}\right.$ being here just the gravitational contribution, $\left.E_{\mathrm{g}} \sim-G M^{2} / R\right)$, are equal but opposite in sign. The system thus exhibits a negative heat capacity, a gain of heat, in an energy balance, producing a decrease of temperature.

This fact is directly related to the long range of gravitation: the virial theorem shows that, for a particle interaction energy $U \propto(\text { distance })^{-k}$, kinetic and total energies have opposite signs for any $k<2$ (Landau and Lifshitz 1960). In addition, the long 
range makes energy non-extensive. In thermodynamics, the value of an additive quantity, for a system, is the sum of values for all its small parts if separated. At given equilibrium conditions, and since volume $i s$ additive, any other additive quantity is extensive: its value is proportional to the volume considered.

Certainly, entropy is additive from its very definition as the logarithm of the product of microstate sets for the small parts of a system (Landau and Lifshitz 1969); we thus wrote $S=\sum_{\alpha} \delta S_{\alpha}$ in section 2. For quantities of mechanical origin, however, one can check the additivity. It clearly holds for, say, number of particles, or momentum, or energy in a body with short-range forces (faraway particles, lying in distinct small parts of a macroscopic system, make negligible force on each other if the energy $U$ follows a power law with $k>2$ ). Charge shielding and overall quasineutrality make energy additive in plasmas, too. On the other hand, gravitation, being always attractive, is radically different: it may admit no lower bound in energy per unit mass, leading, as advanced, to entropy runaway with $E_{\mathrm{M}}$-collapse. (Note that gravitational collapse has been occasionally thought to result in a decrease of entropy! (Terletsky 1971).)

A negative heat capacity means that as heat flows from a hot (part of a) body to a cold one, the first gets hotter and the second colder! This is the gravothermal instability in the so called Antonov model of stellar systems; as in the Triton-Neptune problem the entropy may have no relative maximum to which Antonov's system can go (Lynden-Bell and Wood 1968). The gravothermal instability also makes the core collapse in globular clusters, which may not reach an equilibrium state (Spitzer 1987).

A further thermodynamic paradox of gravitation is the classical Jeans instability: a gas within a box will reach equilibrium, intensive quantities taking uniform values, only if limited in size. For a box with $\left|E_{\mathrm{g}}\right| / E_{\mathrm{i}} \sim G m^{2} N^{2 / 3} /(V / N)^{1 / 3}\left(E_{\mathrm{j}} / N\right)$ large enough (a condition certainly satisfied in the thermodynamic limit, i.e. $N \rightarrow \infty$ with $V / N, E_{\mathrm{i}} / N$ fixed), the gas will locally collapse around perturbations. These are not thermal fuctuations, the Universe being fated to form stellar structures; a 'thermally dead' Universe, being unstable, would not keep dead. Naturally, Newtonian gravitation fails, its field diverging, in the thermodynamic limit. Unsteady, general relativity effects, however, prove to have slowed down, but not quenched, Jeans instability, for conditions throughout most of the Universe history.

\section{Black hole thermodynamics}

As a further consequence of energy non-additivity, stars running out of fuel collapse to thermodynamic 'final' states that depend dramatically on the mass. Low masses end stabilized by electron degeneracy pressure, at low $T / T_{\mathrm{F}}$ ( $T_{\mathrm{F}} \equiv$ Fermi temperature); along the complex and not quite certain route to this dwarf state, and by further radiating as dwarf, the star sheds entropy in addition to (binding) energy and some mass. Equation $\nabla^{2} \phi=4 \pi G \rho$, together with uniform values for $T(\simeq 0)$ and $\mu+m \phi(\rho, \mu$ and $\phi$ being density, chemical and potential and gravitational potential), determine an equilibrium for which the global fundamental relation reads $E_{i}(N, V, S \simeq 0)$, with $N \equiv$ number of nucleons. A condition $p=0$ at the surface provides a second relation, thus yielding a one-parameter family of stellar structures, $E_{\mathrm{i}}(V), N(V)$, or $E(R), M(R)$, with a maximum mass $M_{\max } \sim(\hbar c / G)^{3 / 2} / m^{2}$, at the extreme relativistic limit. Neutron degeneracy pressure, and nucleon interactions, can stabilize somewhat larger masses at much higher densities, and relativistic conditions: since one then has $E_{i} \sim N m c^{2}$ and $G M^{2} / R \sim M c^{2}$, general relativity theory applies, with $E / c^{2}=M \neq N m$.

Apparently, no pressure can prevent a still larger, collapsing mass from reaching its Schwarzschild radius, $R_{\mathrm{s}}(M) \equiv 2 G M / c^{2}$. Purely dynamical results (Misner et al 1973) may then have important consequences on the entropy of such stars. For simplicity we assume zero angular momentum and charge (Schwarzschild black hole):

(i) Once $R$ drops below $R_{s}$ the star collapses all the way to a central singularity, at infinite density and spacetime curvature. From our previous discussions, one expects an unlimited runaway of entropy during collapse. (There are early suggestions of unlimited entropies in general relativity (Tolman 1987).)

(ii) Once $R$ drops below $R_{\mathrm{s}}$, the surface at $R_{\mathrm{s}}$ is an event horizon: a particle or light ray, indeed no signal, can leave its inside (black hole), although they can get in from outside. Thus, entropy generaied during collapse cannot be shed, a fundamental difference with dwarf or neutron stars.

(iii) A bound to the central singularity would arise from quantum effects on space-time curvature at distances of the order of the Planck length, $\left(G \hbar / c^{3}\right)^{1 / 2} \sim 10^{-33} \mathrm{~cm}$. Entropy should then saturate at extremely high values, quantum effects being essential in yielding a definite value for entropy.

(iv) For external observers, a Schwarzschild black hole is characterized by a single parameter, its mass; for instance, the external fields of any initial non-sphericity are wiped out during collapse (Price 1972). One can just write $S_{\mathrm{bh}}(M)$, or $S_{\mathrm{bl}}\left(R_{\mathrm{s}}\right)$.

To determine $S_{\mathrm{bh}}\left(R_{\mathrm{s}}\right)$ recall that, from its very definition, entropy remains additive (even if energy or mass does not) and may be presumed extensive. For observations conducted entirely outside the horizon, the black hole is located at the horizon itself (Misner et al 1973) and so its extension is its area $4 \pi R_{\mathrm{s}}^{2}$. (Although the horizon is a globally 
defined construct of general relativity, with no special significance for local observers, quantum effectsproved essential for the existence of an entropyshould change this. There are suggestions that entropy lies in a sheath around the horizon, its thickness ( $\sim$ Planck length) collapsing as $\hbar \rightarrow 0$, to keep entropy off the black-hole exterior in the classical limit (Wald 1992). Also, quantum effects appear to associate entropy to the global metrics; up to now, the gravitational field, being a macroscopic quantity, had possessed no entropy (Gibbons and Hawking 1977).) We thus have

$$
\begin{aligned}
& S_{\mathrm{bh}} \propto R_{\mathrm{s}}^{2} \propto M^{2} \propto E^{2}, \\
& \frac{1}{T} \equiv \frac{\partial S_{\mathrm{bh}}}{\partial E} \propto E, \quad T S_{\mathrm{bh}}=\frac{1}{2} E .
\end{aligned}
$$

$S_{\mathrm{bh}}$ has been thought non-extensive because of the relation $S_{\mathrm{bh}} \propto M^{2}$ (Landsberg 1992). Extensivity, however, like additivity, is related to volume, mass or energy itself being here non-extensive.

Since only universal constants, in addition to $R_{8}$, or $M$, or $E$, can enter the definite dimensionless expression for $S_{\mathrm{bh}}$ (with $T$ in energy units), one must have

$$
S_{\mathrm{bh}}=\alpha \frac{c^{3}}{G \hbar} R_{\mathrm{s}}^{2}, \quad T=\frac{1}{\alpha} \frac{\hbar c}{4 R_{\mathrm{s}}},
$$

where $\alpha$ is a pure number; $S_{\mathrm{bh}}$ is enormous, as advanced. Also, $T$ being $\hbar$ times a characteristic frequency, one might expect the black hole to radiate as a black body; this has proved to be the case, $\alpha$ coming out to be $\pi$ (Hawking 1975). No such result, however, would follow from a relation $S \propto R_{\mathrm{s}}^{n}$ with $n \neq 2$ !

A number of simple thermodynamic results (Landsberg 1992) follow from (6); in particular, (a) $\mathrm{d} E / \mathrm{d} T$ is negative as expected, and (b) Planck's form of the third law does not hold $\left(S_{\mathrm{bh}} \propto\right.$ $1 / T^{2} \rightarrow \infty$ as $T \rightarrow 0$ ). We note, further, that, at extreme gravitational conditions, purely dynamical results show surprising thermodynamic traces: Bekenstein (1973) first suggested (7) from several such traces in features of black hole dynamics. And a different trace shows up in black holes with non-vanishing angular momentum: when a particle reaches the event horizon it must rigidly rotate with the black hole; this extreme example of the Thirring-Lense, or 'dragging of inertial frames' effect, is also a feature of thermodynamic equilibrium, as recalled in the introduction.

\section{Acknowledgments}

This work was supported by the Comision Interministerial de Ciencia y Tecnología of Spain (ESP890170) and the Departament of Astrophysical, Planetary and Atmospheric Sciences, University of Colorado, during a stay there. I am grateful to Professor M V Goldman for encouragement in this work.

\section{References}

Bekenstein J 1973 Phys. Rev. D 7 2333-46

Binzel R P 1990 Sci. Am. 162(4) 26-33

Callen H B 1960 Thermodynamics (New York: Wiley) ch 13

Darwin G H 1880 Phil. Trans. R. Soc. 171(II) 713-891

Diu B, Guthmann C, Lederer D and Roulet B $1990 \mathrm{Am}$. J. Phys. 58 974-8

Gibbons G W and Hawking S W 1977 Phys. Rev. D 15 2752-6

Goldreich P and Peale S 1966 Astron. J. 71 425-38

Grandy W T, Jr 1987 Foundations of statistical mechanics, I (Dordrecht: Reidel) pp 283-6

Hawking S W 1975 Commun. Math. Phys 43 199-220

Lamb H 1932 Hyarodynamics 6th eda (Cambridge: Cambridge University Press) ch XII

Landau L D and Lifshitz E M 1960 Mechanics (New York: Pergamon) $\$ 10$

1969 Statistical Physics 2nd edn (Oxford: Pergamon) $\S 10$

Landsberg P T 1992 Thermodynamics and Black Holes in Black Hole Physics ed V De Sabbata and Z Zhang (Dordrecht: Kluwer)

Lynden-Bell D and Wood R 1968 Mon. Not. R. Astron. Soc. 138 495-525

Mills B D, Jr 1959 Am. J. Phys. 27 115-7

Misner Ch W, Thorne K S and Wheeler J A 1973 Gravitation (New York: Freeman) ch 32 and 33

Price R H 1972 Phys. Rev. D 5 2419-38

Sanmartín J R, Martínez-Sánchez M and Ahedo E $1993 \mathrm{~J}$. Propul. Power 9 353-60

Spitzer L 1987 Dynamical Evolution of Globular Cluster's (Princeton: Princeton University Press)

Terletsky Ya P 1971 Statistical Physics (Amsterdam: North-Holland) ch VII

Thomson W and Tait P G 1962 Principles of Mechanics and Dynamics-Treatise on Natural Philosophy 1912 edn (New York: Dover) $\$ 276$

Tolman R C 1987 Relativity, Thermodynamics and Cosmology (New York: Dover) § 131

Wald R M 1992 Black Holes and Thermodynamics in Black Hole Physics ed V de Sabbata and Z Zhang (Dordrecht: Kluwer)

Wisdom J, Peale S J and Mignard F 1984 Icarus 58 13752 